\title{
Dislocation Density of Oxygen Free Copper with Compressive Strain Applied at High Temperature
}

\author{
M. Sano ${ }^{1, a^{*}}$, S. Takahashi ${ }^{1, b}$, A. Watanabe ${ }^{1, c}$, A. Shiro, ${ }^{2, d}$ and T. Shobu ${ }^{3, e}$ \\ 1JASRI, 1-1-1 Kouto Sayo-cho, Sayo-gun, Hyogo 679-5198, Japan \\ ${ }^{2}$ QST Quantum Beam Research Directorate 1-1-1 Kouto Sayo-cho, Sayo-gun, Hyogo 679-5148, \\ Japan \\ 3JAEA Materials Sciences Research Center, 1-1-1 Kouto Sayo-cho, Sayo-gun, Hyogo 679-5148, \\ Japan \\ amusano@spring8.or.jp, 'takahasi@spring8.or.jp, 'watanaba@spring8.or.jp, \\ dshiro.ayumi@qst.go.jp, 'shobu@spring8.or.jp
}

Keywords: Dislocation Density, OFC, Plastic Strain, Line Profile Analysis

\begin{abstract}
Dislocation densities of oxygen-free copper (OFC) with compressive strain applied at high temperatures were examined by X-ray line profile analyses with synchrotron radiation. To evaluate the dislocation density, we applied the modified Williamson-Hall and modified Warren-Averbach methods. The dislocation densities of OFC with compressive strain ranging from $0.9-3.8 \%$ were on the order of $1.2 \times 10^{13}-4.2 \times 10^{14} \mathrm{~m}^{-2}$.
\end{abstract}

\section{Introduction}

Oxygen-free copper (OFC) is one of the most popular materials for use in high heat load components that are essential components of many accelerator facilities. At the SPring-8 front-end section, OFC has been incorporated into high heat load components such as photon absorbers and masks in all bending magnet and some undulator beamlines that are subjected to synchrotron radiation with a relatively low power density. Although these components have operated without any thermo-mechanical problems for more than fifteen years, the thermal limitations of OFC should be investigated to deal with increased heat loads in the future, considering the SPring- 8 upgrade plan. Recently, we investigated the fatigue phenomenon of OFC and performed residual strain measurements on the OFC samples by using synchrotron radiation [1, 2]. In addition to these investigations, we have recently been examining the relationship between the plastic strain and the dislocation density for the high heat load materials $[3,4]$.

The purpose of this study is to examine the relationship between the plastic strain and the dislocation density for OFC, as these two parameters are generally correlated with each other. Recently, X-ray line profile analysis has emerged as one of the most powerful methods for the nondestructive investigation of dislocation structures in plastically deformed materials [5]. We examined the dislocation density of OFC samples with compressive strain applied at high temperature. Modified Williamson-Hall and modified Warren-Averbach methods were applied to estimate the dislocation density.

\section{Experimental}

Standard OFC samples, which were of grade C1011 (99.99\% Cu), had known values of compressive plastic strains. The initial configuration of the samples was a cylinder with a height and diameter of $15 \mathrm{~mm}$. These samples were manufactured with compressive plastic strains ranging from $0.9-3.8 \%$ applied at approximately $300^{\circ} \mathrm{C}$ at an approximate strain rate $6.7 \times 10^{-5} \mathrm{~s}^{-1}$. 
The central volumes of the samples, with thicknesses of $2 \mathrm{~mm}$, were cut by electrical discharge machining after the compression stage.

The diffraction experiments were performed at beamline BL02B1 of SPring-8 with a monochromatic beam of $72.3 \mathrm{keV}\left(\lambda=1.72 \times 10^{-2} \mathrm{~nm}\right)$ and a Pilatus $3 \mathrm{X}$ CdTe $300 \mathrm{~K} 2 \mathrm{D}$ detector. Table 1 shows the optical configuration and experimental conditions used for the strain measurements. The strain scanning method with oscillation was used because of the large grain size of the OFC samples. The oscillation length was $\pm 3 \mathrm{~mm}$ in the vertical direction. The measurements were performed at the center of the samples. Fig. 2 shows representative diffraction profiles for the $\mathrm{Cu}$ (200), (311), (400), (331), and (422) reflections of the OFC samples under a compressive plastic strain of $2.6 \%$.

Table 1. Experimental conditions.

\begin{tabular}{c|c}
\hline Beam line & SPring-8/BL02B1 \\
\hline Measurement method & Transmission-type strain scanning method \\
\hline Energy $[\mathrm{keV}]$ & 72.3 \\
\hline Monochromatic crystal & $\mathrm{Si}(311)$ \\
\hline Diffraction angle $(2 \theta)\left[{ }^{\circ}\right]$ & $3.5-13.5$ \\
\hline Camera length $[\mathrm{mm}]$ & 1268 \\
\hline Slit size $($ Width $\times$ Height $)\left[\mathrm{mm}^{2}\right]$ & Divergent Slit $1: 2 \times 0.2$ \\
\hline
\end{tabular}

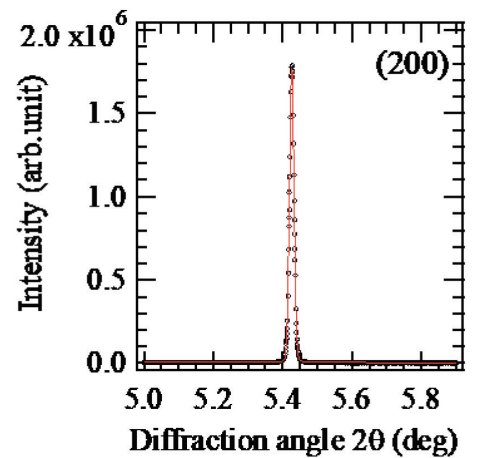

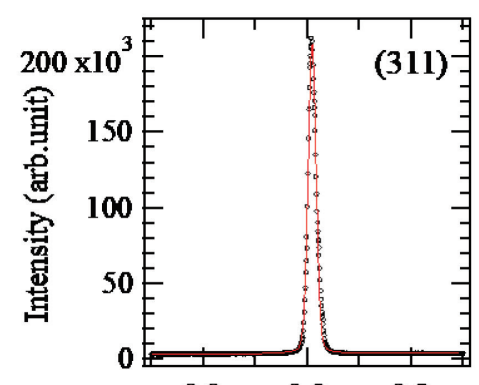

$\begin{array}{llllll}8.7 & 8.88 .9 & 9.0 & 9.1 & 9.2 & 9.3\end{array}$ Diffraction angle $2 \theta(\mathrm{deg})$

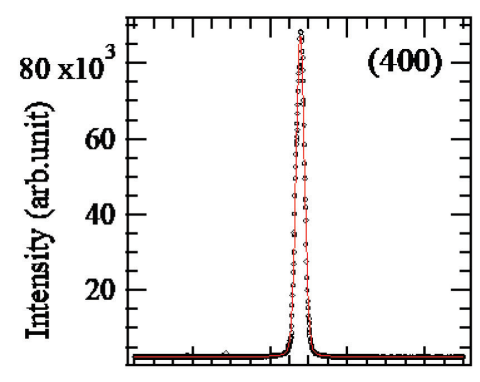

$\begin{array}{llll}10.6 & 10.8 & 11.0 & 11.2\end{array}$ Diffraction angle $2 \theta(\mathrm{deg})$
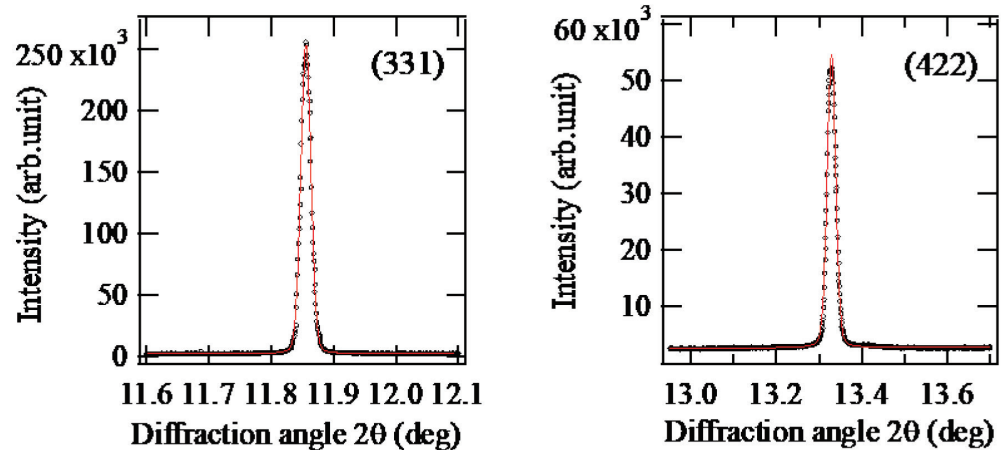

Fig. 1: Cu (200), (311), (400), (331), and (422) diffraction profiles of the OFC sample under a compressive plastic strain of $2.6 \%$. The symbols represent experimental data and the solid line corresponds to the fitting of a pseudo-Voigt function with a linear background. 


\section{Line Profile Analysis}

As shown in Fig. 1, a pseudo-Voigt function with a linear background was applied to the profiles as a fitting function. In this study, it was assumed that instrumental line broadening was negligible, as the instrumental line broadening of the beamline was expected to be less than $0.002^{\circ}$, according to a previous study [5].

The dislocation density was evaluated using the modified Williamson-Hall and modified Warren-Averbach methods, which are based on the FWHM value and the Fourier coefficient of the diffraction profile $[5,6]$. The FWHM values the Fourier coefficients were obtained from the fitting functions. Assuming that the dislocations mainly contribute to the line broadening caused by strain, the modified Williamson-Hall method can be expressed by the following equation:

$$
\Delta K \cong 0.9 / D+\sqrt{\pi M^{2} b^{2} / 2} \sqrt{\rho} K \sqrt{\bar{C}}+O\left(K^{2} \bar{C}\right)
$$

where, $K=2 \sin \theta / \lambda, \Delta K$ is the FWHM, $D$ is the average particle size, $M$ is a constant, $b$ is the absolute value of the Burgers vector, $\rho$ is the dislocation density, $\bar{C}$ is the average contrast factor of the dislocations, and $O$ indicates higher order terms in $K \sqrt{\bar{C}}$. In a cubic crystal system, the average contrast factor can be described as follows:

$$
\bar{C}=\bar{C}_{h 00}\left(1-q H^{2}\right) .
$$

where $\bar{C}_{h 00}$ is the average contrast factor corresponding to the $(h 00)$ reflection, $q$ is a constant, and $H^{2}=\left(h^{2} k^{2}+h^{2} l^{2}+k^{2} l^{2}\right) /\left(h^{2}+k^{2}+l^{2}\right)$. Inserting Eq. 2 into Eq. 1 yields

$$
\left((\Delta K)^{2}-\alpha\right) / K^{2} \cong \beta \bar{C}_{h 00}\left(1-q H^{2}\right) \text {. }
$$

where $\alpha=(0.9 / D)^{2}$ and $\beta=\pi M^{2} b^{2} \rho / 2$. From a linear regression of the left-hand side of Eq. 3 and $H^{2}$, the parameter $q$ can be determined. As shown in Fig. 2, the $q$ values were obtained from the modified Williamson-Hall method for the compressive plastic strains of $0.9 \%$ and $2.6 \%$. The average contrast factor, $\bar{C}_{h 00}$, of copper 0.304 [6] was used in the analysis.
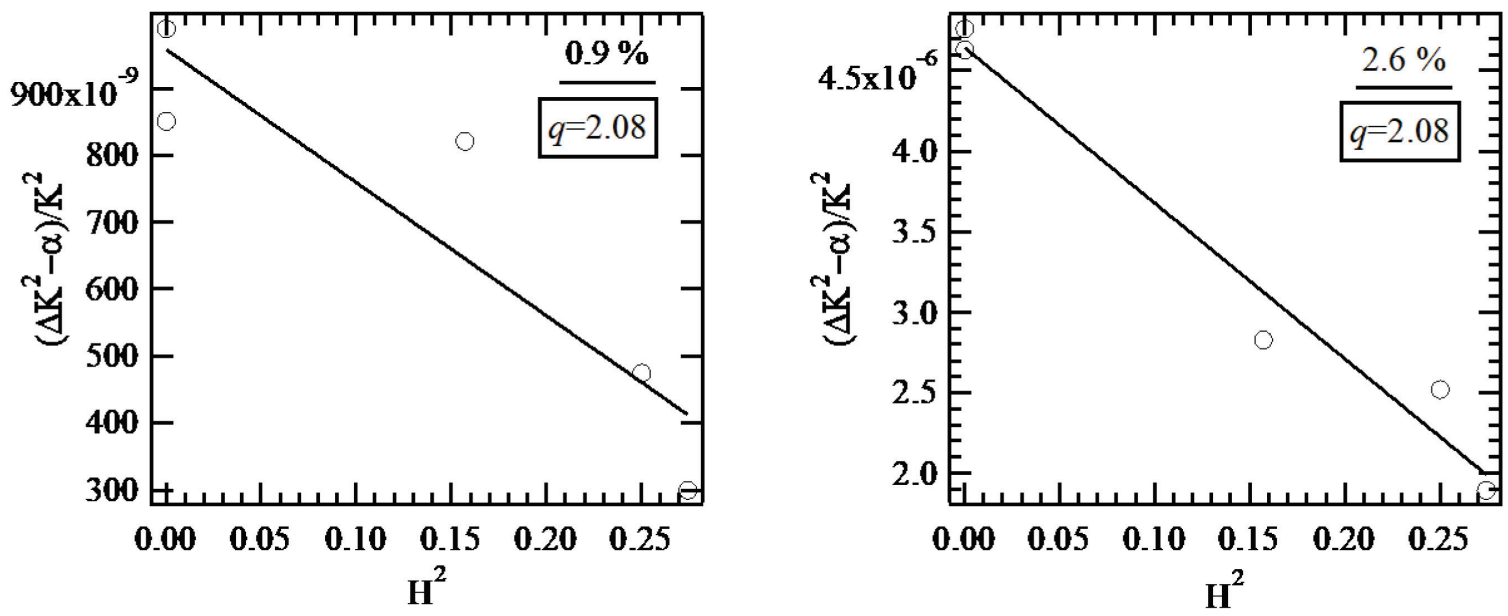

Fig. 2: Relationship between $\left.\left((\Delta K)^{2}-\alpha\right) / K^{2}\right)$ and $H^{2}$ under compressive plastic strains of $0.9 \%$ and $2.6 \%$. The solid line shows the fit of the data to Eq. 3 . 
The dislocation density can be determined from the Fourier coefficients by applying the modified Warren-Averbach method:

$$
\ln A(L) \cong \ln A^{S}(L)-\left(\pi b^{2} / 2\right) \rho L^{2} \ln \left(R_{e} / L\right)\left(K^{2} \bar{C}\right)+O\left(K^{2} \bar{C}\right)^{2} .
$$

where $A(L)$ is the real part of the cosine Fourier coefficient of the diffraction profile, $A^{S}$ is the size Fourier coefficient, $L$ is the Fourier length, $R_{e}$ is the effective outer cut off radius of the dislocation, and $O$ represents higher-order terms in $K^{2} \bar{C}$. By fitting the left-hand side of Eq. 4 as a quadratic function of $K^{2} \bar{C}, A^{S}(L)$ and the slope $(L)=\left(\pi b^{2} / 2\right) \rho L^{2} \ln \left(R_{e} / L\right)$ can be obtained. As shown in Fig. 3, using the $A(L)$, the modified Warren-Averbach method was applied to the compressive plastic strains of $0.9 \%$ and $2.6 \%$. The value of the slope, $X(L)$, for each $L$ value could be determined from the fitting of Eq. 4.
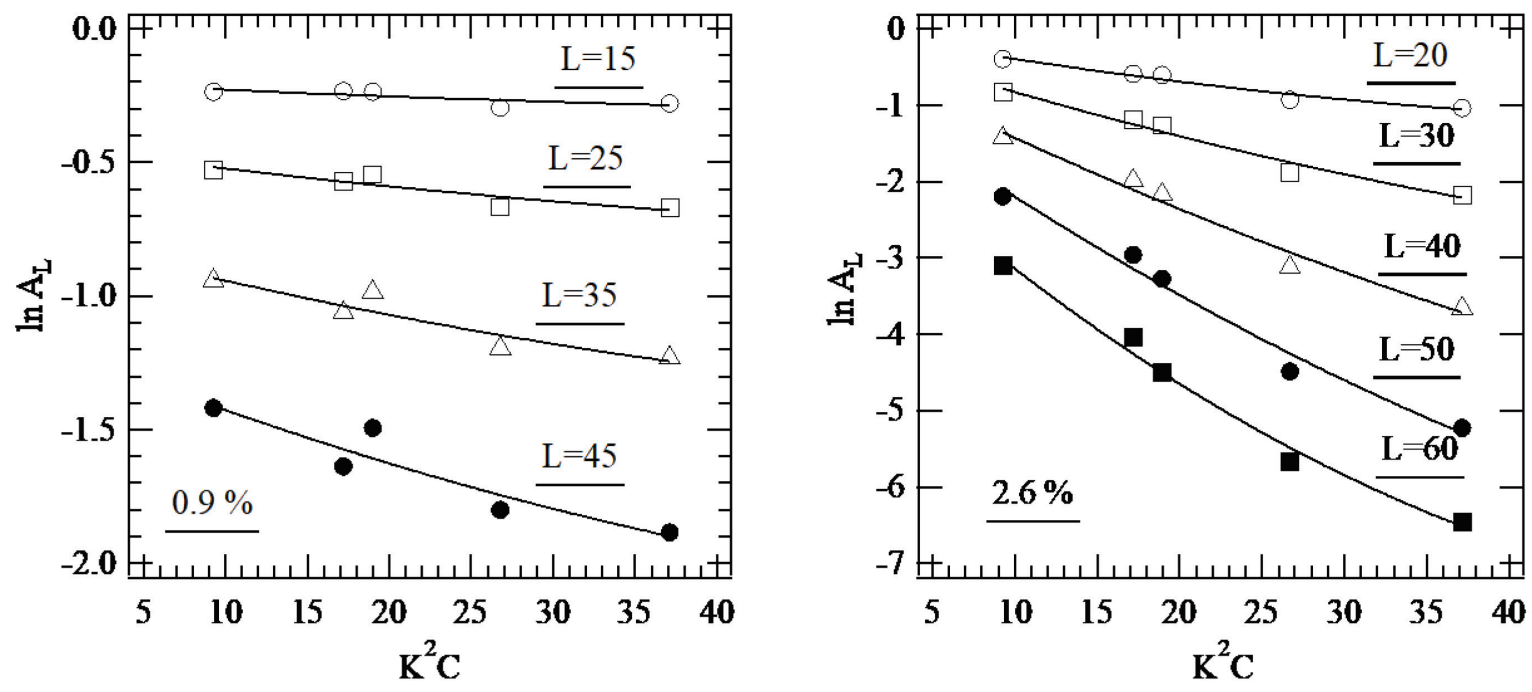

Fig. 3: The relationship between $\ln A_{L}$ and $K^{2} C$ for each $L$ value for compressive plastic strains of $0.9 \%$ and $2.6 \%$. The solid line shows the fit of the data to Eq. 4.

The slope $X(L)$ can then be evaluated according to the following equation:

$$
X(L) / L^{2}=\rho\left(\pi b^{2} / 2\right)\left(\ln R_{e}-\ln L\right)
$$

From the linear regression of $X(L) / L^{2}$ and $\ln L$, the dislocation density, $\rho$, can be obtained. The dislocation densities were evaluated from a linear regression described by Eq. 5, as shown in Fig. 4.

\section{Results and Discussion}

Figure 5 shows changes in the $q$ values with error bars and the dislocation character with theoretical values for each dislocation type, as a function of compressive strain. While half edge half screw dislocations were predominant in the range between 0.9 and $2.6 \%$, the character was pure screw dislocation for $3.8 \%$. Figure 6 shows the dislocation density values with error bars as a function of compressive strain. These error bars were obtained from only the fitting by Eq. 5 . The dislocation density rapidly increased from $1.2 \times 10^{13}$ to $3.6 \times 10^{14} \mathrm{~m}^{-2}$ when the compressive strain increased from 0.9 to $2.6 \%$. On the other hand, the dislocation densities only increased gradually to $4.2 \times 10^{14} \mathrm{~m}^{-2}$, when the compressive strain was changed from 2.6 to $3.8 \%$. 
The dislocation densities of the OFC samples with compressive strain applied at room temperature were $5.1 \times 10^{14}$ and $9.2 \times 10^{14} \mathrm{~m}^{-2}$ for compressive plastic strains of $1 \%$ and $4 \%$, respectively [4]. The values were smaller at $300{ }^{\circ} \mathrm{C}$ than at room temperature within the measured strain range, because it was considered that the mobility of dislocations was higher at high temperature.

As seen in Fig. 5, the error bars of q values were large especially in the case of $0.9 \%$. Improvements will be needed in both the experiment and analysis to obtain more precise values for the dislocation density, since it depends on the dislocation contrast factor.
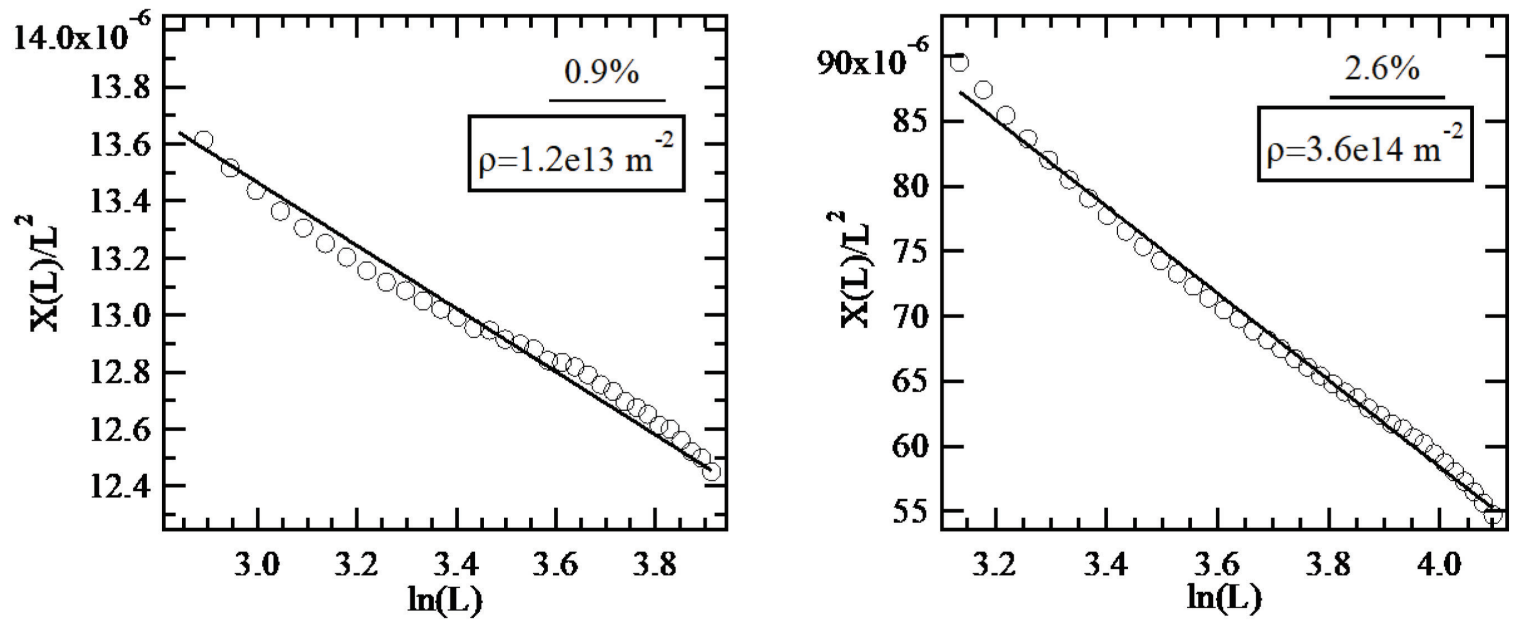

Fig. 4: Relationship between $X(L) / L^{2}$ and $\ln L$ for compressive plastic strains of $0.9 \%$ and $2.6 \%$. The solid lines shows the fits of the data to Eq. 5 .

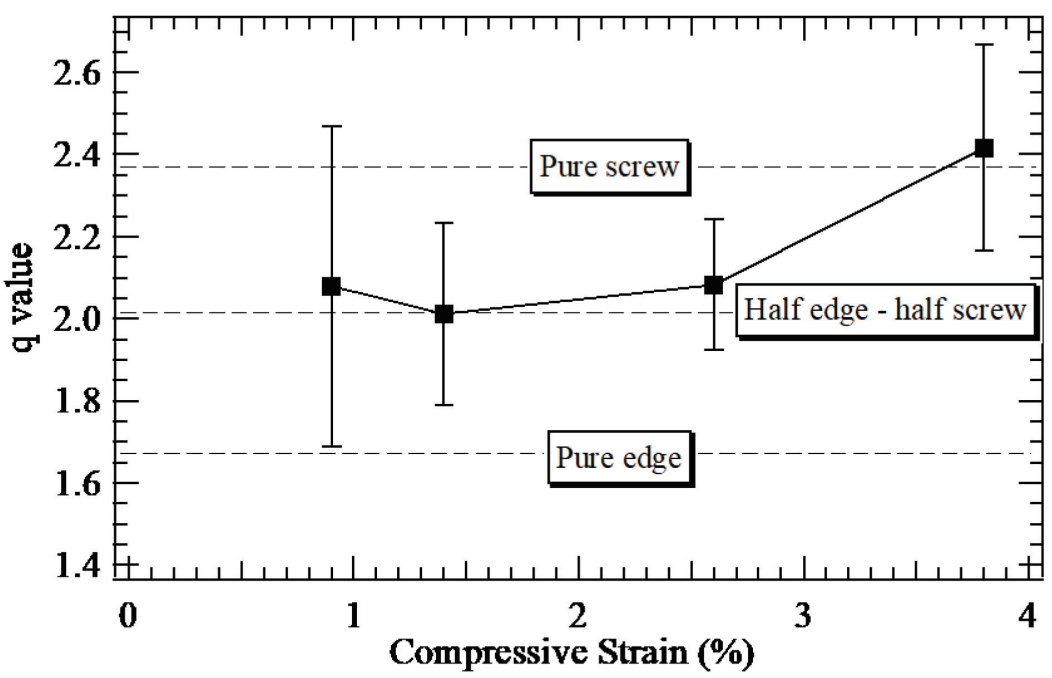

Figure 5: Relationship between the compressive strains and q. The horizontal dotted lines show the theoretical values for each dislocation type.

\section{Summary}

In this study the dislocation densities of OFC samples with compressive strains applied at high temperature were estimated by applying the modified Williamson-Hall and modified Warren-Averbach methods. The dislocation densities and the characters of the dislocations were 
obtained over a strain range of $0.9-3.8 \%$. The dislocation densities of the OFC samples ranged from $1.2 \times 10^{13}$ to $4.2 \times 10^{14} \mathrm{~m}^{-2}$ within the specified strain range.

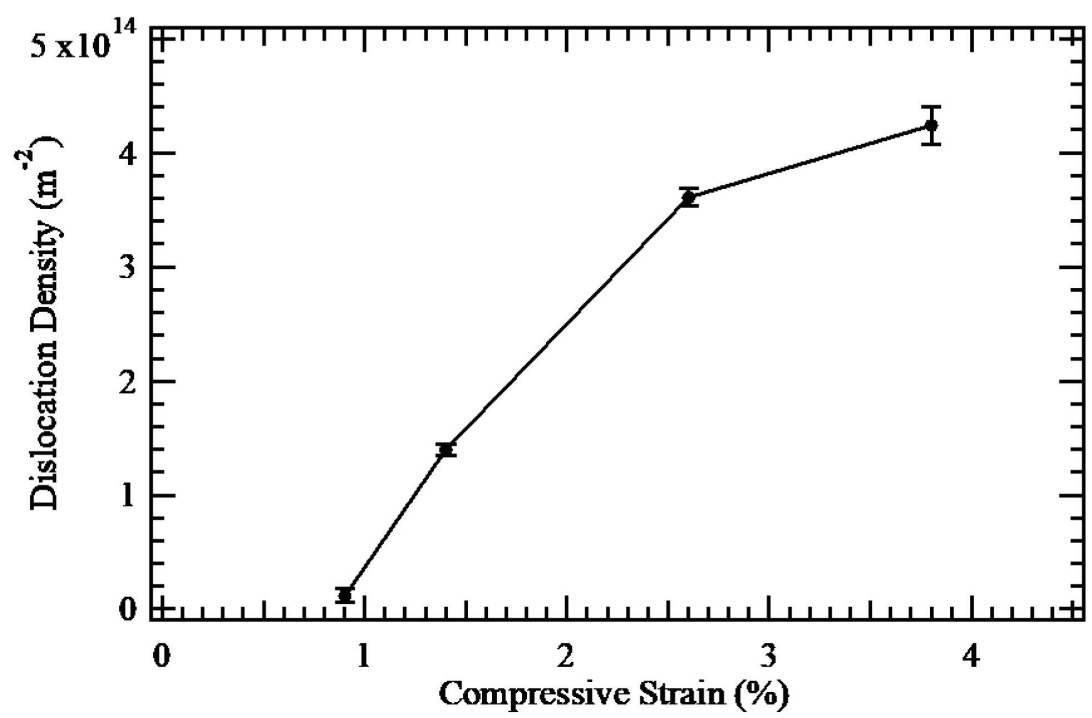

Fig. 6: Relationship between the compressive strain and dislocation density.

\section{Acknowledgements}

The synchrotron radiation experiments were performed at the SPring- 8 with the approval of the Japan Synchrotron Radiation Research Institute (JASRI) (Proposal No. 2016A1335). Use of the modified Williamson-Hall and Warren-Averbach methods developed by Prof. Ungar's work (see references) is acknowledged.

\section{References}

[1] S. Takahashi, M. Sano, A. Watanabe and H. Kitamura, Prediction of fatigue life of high-heat-load components made of oxygen-free copper by comparing with Glidcop, J. Synchrotron Rad. 20 (2013) 67-73. https://doi.org/10.1107/S0909049512041192

[2] M. Sano, S. Takahashi, A. Watanabe, A. Shiro and T. Shobu, Residual strain of OFC using synchrotron radiation, Mat. Sci. For. $777 \quad$ (2014) 225-259. https://doi.org/10.4028/www.scientific.net/MSF.777.255

[3] M. Sano, S. Takahashi, A. Watanabe, A. Shiro and T. Shobu, Dislocation Density of GlidCop with Compressive Strain applied at High Temperature, Mat. Res. Proc. 2 (2016) 609-614.

[4] M. Sano, S. Takahashi, A. Watanabe, A. Shiro and T. Shobu, Dislocation Density of Plastically Deformed Oxygen-Free Copper, Mat. Sci. For. 905 (2017) 60-65. https://doi.org/10.4028/www.scientific.net/MSF.905.60

[5] T. Ungar and A. Borbely, The effect of dislocation contrast on X-ray line broadening: A new approach to line profile analysis, Appl. Phys. Lett. 69 (1996) 3173-3175. https://doi.org/10.1063/1.117951

[6] T. Ungar, I. Dragomir, A. Revesz and A. Borbely, The contrast factors of dislocations in cubic crystals: the dislocation model of strain anisotropy in practice, J. Appl. Cryst. 32 (1999) 992-1002. https://doi.org/10.1107/S0021889899009334

[7] Y. Noda, Current Status of Crystal Structure Analysis BL02B1 Experimental Station, SPring-8 INFORMATION, Volume 02, No.5 (1997) 17-23. 